\title{
La evaluación externa: Un mecanismo para garantizar la calidad de la educación superior en Costa Rica
}

\author{
The External Evaluation: A Mechanism to Ensure the Quality of Higher Education in Costa Rica
}

\section{A avaliação externa: um mecanismo para garantir a qualidade do ensino superior na Costa Rica}

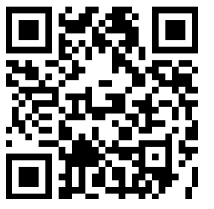

Recibido • Received $\cdot$ Recebido: $13 / 10 / 2016$

Corregido • Revised • Revisado: 08 / 03 / 2018

Aceptado • Accepted • Aprovado: 31/ $03 / 2018$

\footnotetext{
${ }^{1}$ Licenciada en la Enseñanza de la Química, Máster en Psicopedagogía. Académica e investigadora de la División de Educología. Universidad Nacional de Costa Rica.

${ }^{2}$ Doctora en Ecología Marina, académica e investigadora de la Escuela de Ciencias Biológicas, Universidad Nacional de Costa Rica.

${ }^{3}$ Licenciada en Enseñanza de las Ciencias, Máster en Docencia Universitaria. Académica e investigadora de la División de Educología y la Escuela de Ciencias Biológicas. Universidad Nacional de Costa Rica.
} 
doi: http://dx.doi.org/10.15359/ree.22-2.16

URL: http://www.una.ac.cr/educare

CORREO: educare@una.cr

Resumen: El objetivo de este estudio es evidenciar que la acreditación conlleva un proceso dinámico y complejo que permite el mejoramiento de la educación superior, debido a que asegura y promueve la calidad en todos los aspectos que conforman este nivel educativo. En este artículo de revisión bibliográfica, se consideraron criterios asociados a diferentes experiencias realizadas tanto en Costa Rica como en otros países latinoamericanos en relación con las normas utilizadas por los entes queacreditan para que se realicen las autoevaluaciones. Además, se hace una reflexión sobre los distintos mecanismos empleados para la evaluación de la calidad en las instituciones de educación superior, así como el papel de la acreditación en estas instituciones versus el aseguramiento de la calidad y como propiciar el desarrollo de una cultura institucional hacia los procesos de calidad en Costa Rica. Entre los principales resultados se encontró que estos procesos arrojan las fortalezas y debilidades que tienen las carreras o instituciones que se someten a este proceso, lo que permite la aplicación de un plan de mejoras conducente al mejoramiento continuo que está sometido a verificación externa, según criterios y normas de calidad establecidos.

Palabras claves: Educación superior; acreditación; calidad educativa; evaluación educativa.

Abstract: This study aims to show that the accreditation involves a dynamic and complex process. The accreditation allows the improvement of higher education because it ensures and promotes quality in all the aspects that compose this educational level. This bibliographic review article considered a range of criteria concerning the standards accrediting bodies use to have self-assessments made. These criteria are associated with different experiences carried out both in Costa Rica and in other Latin American countries. In addition, this article proposes a reflection on the different mechanisms used to evaluate the quality in higher education institutions, as well as an analysis on the role of accreditation in these public universities versus the quality systems and the ways to promote the development of an institutional culture that leads to quality processes in Costa Rica. Among the main findings, the study showed that these processes put in evidence the strengths and weaknesses of the careers or institutions that undergo the accreditation, which allows the implementation of improvement plans leading to a continuously improving process that is subject to external verification following established criteria and standards.

Keywords: Higher education; accreditation; educational quality; educational evaluation.

Resumo: O objetivo deste estudo é demonstrar que a acreditação envolve um processo dinâmico e complexo que permite a melhoria do ensino superior, pois garante e promove a qualidade deste nível educativo em todos os aspectos. Neste artigo de revisão bibliográfica, foram considerados critérios associados a diferentes experiências, tanto na Costa Rica quanto em outros países da América Latina, em relação as regras utilizadas pelas entidades que aprovam a realização das autoavaliações. Além disso, faz-se uma reflexão sobre os diferentes mecanismos utilizados para a avaliação da qualidade nas instituições de ensino superior, bem como o papel da acreditação nessas instituições versus a garantia da qualidade, e formas de fomentar na Costa Rica o desenvolvimento de uma cultura institucional voltada aos processos de qualidade. Entre os principais resultados, constatou-se que esses processos evidenciam os pontos fortes e fracos das carreiras ou instituições que passam por esse procedimento, o que permite a aplicação de um plano de aperfeiçoamento, levando à melhorar continuamente $o$ que está sujeito a verificação externa, segundo critérios e padrões de qualidade estabelecidos.

Palavras-chave: Ensino superior; acreditação; qualidade educacional; avaliação educacional 


\section{Introducción}

Es común encontrar que los procesos de autoevaluación de las carreras de enseñanza superior en el país están asociados también a procesos de acreditación o certificación de la calidad y pareciera que Costa Rica, al igual que otros países, ha optado por este modelo de rendición de cuentas a un ente externo, en aras de asegurar que se genere la cultura del mejoramiento continuo. En nuestro caso, este ente es el Sistema Nacional de Acreditación de la Enseñanza Superior (SINAES), el cual propone un proceso técnico destinado a asegurar y promover la calidad de los programas de estudio de las distintas carreras que imparten las instituciones de educación superior, mediante la aplicación de mecanismos de autoevaluación y verificación externa (Jara, 2011).

Estos mecanismos de autoevaluación que son el eje central de los procesos de acreditación permiten conocer los comportamientos, tendencias y configuraciones existentes de la realidad investigada y conlleva a encontrar particularidades y diferentes aspectos que ayudan a construir un panorama de la situación existente, con el fin de la mejora continua en aras de la calidad (Agencia Nacional de Evaluación y Acreditación de la Educación Superior [ANEAES], 2014).

Es decir, el objetivo central de la acreditación para la enseñanza superior es promover y estimular el continuo mejoramiento de las carreras, según criterios y normas de calidad establecidos por los entes acreditados para ese fin, en nuestro caso SINAES. De ahí que la acreditación universitaria se concibe como un recurso mediante el cual las carreras garantizan que los servicios que ofrecen son de calidad.

En esta misma línea, los informes de autoevaluación de diferentes carreras de la Universidad Nacional -entre ellos el Informe de autoevaluación de la carrera Licenciatura en la Enseñanza de las Ciencias (Universidad Nacional, Escuela de Ciencias Biológicas, 2013) y el Informe de autoevaluación de la carrera Bachillerato en Biología con énfasis en Biología Tropical, en Biología Marina y en Biotecnología; Licenciatura en Biología con énfasis en Manejo de Recursos Naturales y en Manejo de Recursos Marinos y Dulce Acuícolas (Universidad Nacional, Escuela de Ciencias Biológicas, 2014) - han permitido conocer las fortalezas y debilidades de esas carreras y han dado pie a la elaboración de un plan de mejoras sustentado en estándares de calidad dictados por el SINAES (2009), que ha concentrado esfuerzos y recursos institucionales para solucionar, de acuerdo con las posibilidades, dichas debilidades. Esto ha traído beneficios en infraestructura, en la gestión académica, en la actualización del profesorado, en la mejora de la ejecución de lineamientos, en el seguimiento a estudiantado graduado, en la revisión y actualización de los planes de estudios, entre otros aspectos.

A la vez, cabe destacar que la autoevaluación, la evaluación interna, la evaluación externa, así como la elaboración y ejecución de los planes de mejora, son opciones que permiten el aseguramiento de la calidad de los diferentes procesos académicos trayendo también beneficios. Además, en el contexto actual, la creciente demanda y complejidad de formación 
doi: http://dx.doi.org/10.15359/ree.22-2.16

URL: http://www.una.ac.cr/educare

CORREO: educare@una.cr

de profesionales ha masificado, en algunos casos, la oferta y, como consecuencia, no siempre se cuenta con la rigurosidad y excelencia que se espera de la educación superior, por lo que estos procesos contribuyen a subsanar, en parte, esas debilidades.

En este artículo se tratarán aspectos medulares como los mecanismos para la evaluación de la calidad en la educación superior, la acreditación versus el aseguramiento de la calidad, así como la importancia y el desarrollo de una cultura institucional hacia los procesos de calidad, con el fin de dar a conocer los beneficios de los procesos de autoevaluación como mecanismos que ayudan al mejoramiento de la educación superior en Costa Rica.

\section{Desarrollo}

\section{Mecanismos para la evaluación de la calidad en la educación superior}

Los procesos y mecanismos de acreditación se han difundido en muchos países, con el interés de mejorar las diferentes ofertas académicas y Costa Rica no es la excepción, por el contrario, desde 1992 CONARE tomó el acuerdo de la creación del Sistema Nacional de Acreditación de la Educación Superior (SINAES) y, en 1997 fue ratificado. Desde entonces, tanto universidades del sector público como privado han apostado por la acreditación de sus carreras, aumentando el número de carreras acreditadas, año con año. Además, es indiscutible que estos procesos son beneficiosos para la gestión y mejoramiento de la calidad en este nivel educativo. También en Latinoamérica existen iniciativas regionales, desde hace varios años, tales como el mecanismo de reconocimiento mutuo de los procesos de acreditación y de la validez académica de títulos en el Mercado Común del Sur (MERCOSUR), llamado Sistema de Acreditación de Carreras Universitarias para el Reconocimiento Regional de la Calidad Académica de las Respectivas Titulaciones (ARCU-SUR), y las agencias regionales especializadas creadas en Centroamérica para la acreditación de carreras de Ingeniería y Arquitectura, así como carreras del área agroalimentaria y programas de posgrado (Pires y Lemaitre, 2008).

En esta misma línea, en el informe Situación educativa de América Latina y el Caribe: Hacia una educación de calidad para todos, presentado por la UNESCO (2013), explica que, en América Latina, las políticas para la educación superior intentan construir sistemas de acreditación y aseguramiento de la calidad, conducentes a una gestión coordinada de las instituciones que cada vez se tornan más heterogéneas. Esto hace necesaria la creación de nuevas instituciones encargadas, entre otros aspectos, de asegurar su calidad y promover en ellas una cultura de la información, del complimiento, la transparencia y la responsabilidad.

En el contexto actual, la idea de calidad se convirtió en un instrumento indispensable para la evaluación de programas e instituciones de educación superior. Según Scheele (2009), los modelos tradicionales e informales de autorregulación académica que fueron considerados durante mucho tiempo como medios efectivos para garantizar la calidad, han sido sustituidos 
por mecanismos formales de aseguramiento de la calidad que conllevan varios procedimientos externos de evaluación e inspección.

En este sentido, es urgente incorporar en las instituciones, tanto nacionales como regionales, las disposiciones pertinentes que extiendan la competencia de nuestros sistemas a la acreditación de los programas (Tünnermann, 2008). Es importante destacar que Costa Rica ha venido haciendo esfuerzos por adherirse a iniciativas regionales, tal es el caso de la adhesión a la OCDE en junio de 2016, que puso en marcha el Programa Regional para América Latina y el Caribe (LAC). Esta entidad elaboró un informe acerca de las políticas para educación en Costa Rica, en el que, entre otros aspectos, le dice que "el sistema de educación universitaria necesita de una extensa reforma en aseguramiento de calidad, financiamiento y gobernanza para garantizar su apoyo a las metas de desarrollo de Costa Rica" (OCDE, s. f., p. 7).

La evaluación institucional, como parte del Sistema Nacional de Acreditación de la Enseñanza Superior, es un procedimiento científico que se rige estrictamente por parámetros de calidad establecidos internacionalmente, que además son acordados y aceptados por la comunidad académica de cada país.

[En este sentido] la evaluación institucional se sustenta en principios de calidad que guían su accionar en el proceso evaluativo, así como en normas y técnicas que direccionan los procedimientos a ser aplicados para recoger información válida, pertinente, relevante, útil y oportuna para el diagnóstico de la situación presente y la proyección de las estrategias necesarias para la mejora. (ANEAES, 2014, p. 19)

Para ello deben existir parámetros que aseguren la calidad de los diferentes programas que estas ejecutan y de ahí que los sistemas de acreditación trabajan en el logro de lineamientos dirigidos al mejoramiento continuo de procesos amparados a estándares internacionales, porque, según Tünnermann (2008), "en el contexto de la sociedad contemporánea, las instituciones de educación superior son parte del mundo académico global, [por lo que] hoy en día, ninguna universidad puede sustraerse de las relaciones internacionales y los intercambios académicos con el extranjero" (p. 313).

Por otra parte, las universidades deben responder a las necesidades de desarrollo de un país y su funcionamiento debe estar en concordancia con las exigencias sociales y de conocimiento que requiere una nación para ser competitiva en un mundo globalizado. De ahí la importancia de promover los mecanismos necesarios para asegurar el mejoramiento de la calidad de las ofertas académicas, ya que el desarrollo y la calidad de vida de un país están muy ligados a la calidad de su sistema educativo y, en especial, al nivel de la educación superior que debe garantizar la formación necesaria de profesionales para que aporten las bases que permitan el mejoramiento de un país (Padilla-Canales, Brooks-Calderón, Jiménez-Porras y Torres-Salas, 2015). 
doi: http://dx.doi.org/10.15359/ree.22-2.16

URL: http://www.una.ac.cr/educare

CORREO: educare@una.cr

Según Miranda (2007), las universidades segeneraron como un medio para institucionalizar la educación, la investigación y la extensión, con el fin de formar profesionales de alta calidad en diferentes campos, que aporten saberes, que preserven la riqueza cultural y científica de la sociedad por medio de la reflexión crítica y, a la vez, enriquezcan con nuevas ideas. Sin embargo, para asegurar profesionales de calidad, es necesario también desarrollar procesos de autoevaluación que permitan dar seguimiento a la calidad con la que se están ejecutando los diferentes planes de estudio en las instituciones de educación superior.

La acreditación, como forma de asegurar la calidad de la educación superior, se viene poniendo en práctica en Costa Rica desde finales de los 90, después de la creación de SINAES. Además, la acreditación es una forma de asegurar la calidad en la actualidad, donde la apertura y autonomía creciente de instituciones de nivel superior no siempre cumple con niveles de rigurosidad en los programas ofertados para diferentes carreras. En este sentido, Scheele (2009) menciona que la calidad se convierte en un criterio cada vez más importante para que el estudiantado y personal académico puedan establecer cuáles instituciones de educación superior merecen su preferencia.

Existen varios tipos de acreditación, dependiendo del sistema educacional de cada país y del objetivo de los procesos de acreditación. En Costa Rica, como ya se ha mencionado, SINAES es el ente encargado de la acreditación de aquellas carreras que así lo solicitan, para lo cual se aplica un proceso que considera la evaluación de aspectos administrativos, de docentes, del proceso de admisión, de estudiantes, de la infraestructura, de los servicios estudiantiles, de estudiantado graduado y de entes empleadores; en fin, es una lectura de la realidad de la carrera tanto interna como externamente.

Este proceso para la acreditación de las carreras de educación superior conlleva, generalmente, las siguientes etapas (SINAES, 2009):

- Etapa de autoevaluación: Realizada por una comisión de personal académico de la institución o carrera, quienes redactan un informe en el que se plasman los resultados obtenidos de una serie de instrumentos (que contienen estándares y criterios establecidos por el ente acreditador) que se aplican a diferente actores (estudiantes, personal académico y administrativo, estudiantado graduado, y entes empleadores), así como la ejecución de talleres a diferentes poblaciones involucradas con la carrera. Este informe, una vez avalado por las autoridades de la institución, es enviado al ente acreditador, conjuntamente con un plan de mejoramiento como resultado de la autoevaluación.

- Etapa de visita de pares externos:El programa, carrera o institución recibeen las instalaciones al equipo evaluador externo designado por el ente que certifica la acreditación, el cual, después de reunirse con diferentes poblaciones y contrastar la información escrita con la realidad, genera un informe que incluye una recomendación al organismo acreditador. 
- Etapa de estudio por parte del organismo de acreditación: El informe generado por el equipo evaluador externo le permite tomar una decisión final, que se comunica a la universidad correspondiente y a todos los sectores interesados. De ser positivo, se establece un tiempo cuando la carrera queda acreditada con la responsabilidad de ejecutar un plan de mejoras en el plazo por el que fue acreditado, con el fin se subsanar

- Etapa de reacreditación: Cuando el periodo por el que fue acreditada la carrera termina, el proceso de acreditación comienza nuevamente, podría decirse que es un proceso cíclico, para ello se valora el cumplimiento del plan de mejoras, así como debe hacerse nuevamente una autoevaluación y generar un nuevo informe que es avalado por las autoridades de la institución presentado nuevamente al ente acreditador.

También existe la posibilidad de que una institución, luego del proceso de autoevaluación, no logre cumplir con los estándares de calidad, en este caso el ente acreditador le otorga un período que generalmente es de un año para que ejecute un plan de mejoras que le permita cumplir con los estándares requeridos.

\section{Acreditación en las instituciones de educación superior versus aseguramiento de la calidad}

Uno de los aspectos más importantes de toda sociedad es la educación y formación integral de su ciudadanía, más aún en la educación superior, donde se forman profesionales para el servicio y bien de la sociedad. Por ello, las universidades han sido sometidas a procesos de acreditación, con el fin de mantenerse vigentes y demostrar a los sectores sociales la congruencia de sus funciones, oferta educativa y, con esto, su calidad.

Según el artículo 11 de la Declaración Mundial sobre la Educación Superior_en el siglo XXI. Visión y acción (UNESCO, 1998), la calidad de la educación debe ser pluridimensional y dinámica, es decir, que componentes como el modelo educativo, la misión, los objetivos de la universidad, la infraestructura, así como sus grupos de interés: estudiantes, personal académico, entes empleadores, la sociedad y el gobierno deben estar interrelacionados. Además, se debe garantizar una dimensión internacional que permita el intercambio de conocimientos, por ende, la calidad de la educación no se enmarca a una sola instancia; por el contrario, debe ser un proceso íntegro que demanda reflexión, diálogo y esfuerzo conjunto.

Para obtener una calidad educativa, las universidades deben garantizar que los intereses académicos toman prioridad sobre otros intereses y que estos son ejercidos con integridad, además de exigencia académica, recursos adecuados, eficacia de docentes con suficiente preparación en las áreas que enseñan, además han de someterse a procesos internos y externos para evaluar la calidad de sus carreras y programas de estudio, con el fin demostrar que se alcanzan la metas educativas (Pedraja, 2013). 
doi: http://dx.doi.org/10.15359/ree.22-2.16

URL: http://www.una.ac.cr/educare

CORREO: educare@una.cr

Los sistemas de acreditación son los entes encargados de certificar y asegurar que la educación que brindan las universidades sea de alta calidad y que los programas de estudios impartidos en las diferentes carreras sometidas a estos procesos se adaptan a las necesidades de la sociedad y permiten alcanzar el éxito a las poblaciones egresadas, así como los niveles de formación y destrezas necesarias de la profesión (Yamada, Castro y Rivera, 2012); igualmente, la verificación que realizan los pares evaluadores ayuda a identificar las debilidades y, con ello, establecer criterios mediante un compromiso de mejoras que garanticen la excelencia y, por ende, los títulos otorgados tengan mayor reconocimiento y aceptación.

De acuerdo con la experiencia en procesos de acreditación de la UNA y como se desprende de los informes de diferentes carreras, tales como: Enseñanza de las Ciencias (Universidad Nacional, Escuela de Ciencias Biológicas, 2013) y de la carrera de Biología (Universidad Nacional, Escuela de Ciencias Biológicas, 2014), entre otros, que la acreditación ha traído beneficios en la infraestructura, en la gestión académica, en la actualización del profesorado, en la mejora de la ejecución de lineamientos, en el seguimiento a su estudiantado graduado, así como en la revisión y actualización de los planes de estudios.

Entre los aspectos que, en términos generales, se mejoraron según el Informe de autoevaluación de la carrera Licenciatura en la Enseñanza de las Ciencias (Universidad Nacional, Escuela de Ciencias Biológicas, 2013) y el Informe de autoevaluación de la carrera Bachillerato en Biología con énfasis en Biología Tropical, en Biología Marina y en Biotecnología, Licenciatura en Biología con énfasis en Manejo de Recursos Naturales y en Manejo de Recursos Marinos y Dulce Acuícolas (Universidad Nacional, Escuela de Ciencias Biológicas, 2014) están: los procesos de admisión se han agilizado, así mismo los mecanismos de divulgación e ingreso a las carreras en igualdad de oportunidades, además que el estudiantado está mejor informado acerca de la vida universitaria y la carrera que cursa. En lo que respecta a la correspondencia con el contexto, es importante destacar que en los programas de los cursos de diferentes planes de estudio se han incorporado temáticas y actividades académicas que fomentan la actualización en el avance y desarrollo de diferentes disciplinas. En esta misma línea se han incrementado la cantidad de vínculos de las carreras con la correspondiente comunidad académica, con el fin de mejorar el intercambio, la realimentación y generación de propuestas de solución a problemas del contexto.

En esta misma línea, los informes mencionados (Universidad Nacional, Escuela de Ciencias Biológicas, 2013; Universidad Nacional, Escuela de Ciencias Biológicas, 2014) arrojan que, en relación con los planes de estudio, se han desarrollado acciones tales como actividades orientadas al personal docente de las diferentes carreras, para que tengan un conocimiento profundo de los respectivos planes de sus carreras, se han actualizado los referentes universales y las corrientes del pensamiento que los fundamentan, con el fin de lograr una mejor comprensión y puesta en práctica del quehacer docente. También se ha analizado la secuencia de los cursos, los requisitos y correquisitos establecidos en las mallas curriculares de diferentes carreras, trayendo un mejoramiento de la oferta, además se incorporaron contenidos de ética 
tanto para el manejo de temas en específico, como para el ejercicio profesional. En otros casos se realizaron rediseños de planes de estudios, tomando en cuenta recomendaciones hechas por personas graduadas, entes empleadores, estudiantes y personal académico, entre otros. Estos generaron propuestas actualizadas y pertinentes en la formación de profesionales con mejor preparación para contribuir con el desarrollo del país.

También se observan acciones dirigidas a que existan mecanismos de evaluación que permitan la retroalimentación y mejora del personal administrativo de diferentes unidades académicas, elemento muy importante en la gestión de los diferentes planes de estudios.

En cuanto a infraestructura, se observan acciones en pro de la mejora de las plantas físicas de los edificios que albergan las distintas carreras, tomando en cuenta las condiciones estipuladas por la Ley 7600 (Asamblea Legislativa de la República de Costa Rica, 1996). Ello ha dado como resultado que personas con condiciones especiales tengan acceso y puedan estudiar. También se han llevado a cabo importantes cambios en aulas, laboratorios, oficinas, bibliotecas, aceras, rampas, entre otros espacios; se han implementado, a la vez, normas de seguridad, higiene y salud ocupacional, que propician mejores condiciones para desarrollar actividades académicas y extracurriculares en los diferentes espacios requeridos.

Los centros de información y recursos, así como las bibliotecas, se han fortalecido y brindan servicios más eficientes y oportunos. Además, con el objetivo de que exista un mayor alcance de la información, se han reforzado los recursos en línea disponibles en las universidades.

Otro aspecto que se ha visto beneficiado con las acreditaciones es el referente al aumento de recursos para la compra de equipo y materiales, permitiendo que los profesorados puedan hacer uso de equipos y tecnologías de la comunicación que apoyen el proceso educativo.

Así mismo, el proceso educativo se ha visto beneficiado con acciones conducentes a mejorar las interacciones entre docentes, y sus responsabilidades con el estudiantado, favoreciendo el análisis y la integración del conocimiento en función de la carrera acorde con la realidad nacional e internacional. Además, se ha impulsado la evaluación docente con el fin de que exista una retroalimentación a la docencia y que, a la vez, permita un proceso de actualización continua al profesorado, en función de enriquecer los conocimientos en las propias disciplinas y en la pedagogía universitaria.

Por otra parte, la investigación se ha favorecido con la definición de líneas acordes con la realidad costarricense y foránea. Ha permitido el enriquecimiento en este campo, que es esencial en el quehacer de las universidades, ya que propicia el estar a la vanguardia en la producción de conocimiento. También ha contribuido en la creación de insumos educativos a partir de las investigaciones de los cuerpos docentes de las carreras y ha generado apoyo para el desarrollo de los cursos. De igual manera, la acreditación ha dado un impulso importante a la extensión, con acciones dirigidas a fortalecer y promover esta actividad en beneficio del país, ya que se trabaja directamente con diferentes comunidades y se ayuda, así, a la inserción social. 
doi: http://dx.doi.org/10.15359/ree.22-2.16

URL: http://www.una.ac.cr/educare

CORREO: educare@una.cr

En esta misma línea, el desempeño docente, la interacción con el estudiantado graduado y la proyección de las carreras también se han visto favorecidas con la implementación de los procesos de acreditación, ya que se ejecutan estrategias para garantizar una educación contextualizada, que permita un desarrollo integral del estudiantado, lo cual se evidencia en personas graduadas satisfechas con su formación y en entes empleadores que dan referencia de la calidad de profesionales que salen al mercado.

De acuerdo con las mejoras señaladas, es evidente que los procesos de acreditación redundan en un claro beneficio del mejoramiento de las condiciones necesarias de las instituciones de educación superior; permiten, así, la calidad en los procesos de formación de profesionales con la debida preparación o capacitación para enfrentarse de manera efectiva en distintas situaciones del mundo laboral, con el fin de contribuir al desarrollo del país y la transformación de la realidad.

Sin embargo, para mantener esa calidad, es necesario que las universidades se evalúen continuamente para determinar si están alcanzando sus objetivos, además deben velar porque se ejerzan funciones estratégicas para el desarrollo cultural, científico y tecnológico, como espacio de reflexión y creatividad, que aporte las herramientas necesarias para el análisis social, la reflexión crítica y la sostenibilidad como instituciones sociales (Zapata y Tejeda, 2009). En síntesis, estas evaluaciones continuas permiten retroalimentar las carreras, para el desarrollo pertinente de sus profesionales.

\section{El desarrollo de una cultura institucional hacia los procesos de calidad en Costa Rica.}

Los procesos de acreditación en las universidades costarricenses, tanto públicas como privadas, han generado un debate hacia el fortalecimiento de la calidad, no solo de unas cuantas carreras, sino de toda la oferta académica que se brinda. La abrumadora realidad y proliferación de las llamadas "universidades de garaje" ha puesto en la mesa de discusión cuán oportuno e importante es garantizarle al estudiantado universitario, ya sea desde las instituciones universitarias estatales o desde las universidades privadas, una formación profesional de calidad, la cual, en el panorama costarricense, se obtiene mediante la acreditación de carreras.

Sin embargo, llegar a una cultura institucional hacia la calidad en la educación superior de Costa Rica es un proceso que aún está en desarrollo, ya que, si bien SINAES ha construido todo un aparato institucional para el proceso de evaluación externa de la calidad de la educación universitaria y las universidades estatales están direccionadas a buscar este mejoramiento; la incursión en el proceso de autoevaluación con miras a la acreditación es una herramienta que no todas las unidades académicas dentro de las instituciones de educación superior quieren o pueden asumir, dado que implica cambios académicos y administrativos que pueden generar reticencia en algunas poblaciones involucradas, que pueden tener aversión a ser fiscalizadas por un ente externo. Según Águila (2005), en muchos casos para las carreras universitarias 
en Latinoamérica, la rendición de cuentas ante un ente externo a la universidad es un punto de inflexión y acatamiento que en la mayoría de ocasiones constituye el principal "pero" para introducir la acreditación de una carrera. La condición de institución autónoma con reglamentos y normativas propias es otro punto medular en las dificultades que surgen como excusa para el mejoramiento académico institucional (Giraldo, Abad y Díaz, s. f.).

Para llegar a que los procesos de acreditación de las carreras universitarias sean parte de la cultura institucional, es fundamental que dicho proceso sea aceptado e interiorizado por los primeros eslabones del engranaje universitario, es decir, estudiantes, docentes y personal administrativo de una determinada carrera (Torche, Martínez, Madrid y Araya, 2015). Si no hay un compromiso de dichos actores por el mejoramiento de la carrera, será muy difícil que el proceso avance, ya que estos grupos son los principales ejecutores de los compromisos de mejoras adquiridos una vez se obtiene la acreditación.

Si hay un total compromiso a lo interno de una unidad académica para acreditar una carrera, el siguiente peldaño en afianzar y comprometerse con la calidad deben ser las instancias administradoras de una universidad, lo que fundamentaría el compromiso institucional por un mejoramiento real de las carreras ofrecidas. Dentro de la institucionalidad propia de cada ente universitario, los procesos de calidad académica fortalecen una serie de acciones que enriquecen no solo a las carreras acreditadas, sino también a la institución. Según SINAES (2015), la acreditación trae beneficios como el mejoramiento de la calidad no solo de la carrera acreditada, sino también se permea a toda la universidad con el "compromiso de calidad y mejora continua". Además, se fortalecen y amplían los vínculos externos que repercuten directamente en la enseñanza.

Un punto medular de los procesos de calidad en la enseñanza universitaria consiste en que, una vez que una carrera inicia con la acreditación, hay un conocimiento que genera sinergia hacia otras carreras, enriqueciendo y promoviendo el desarrollo de mecanismos de mayor eficiencia académica y administrativa en las universidades. Asimismo, esta sinergia se ve favorecida por una demanda externa de profesionales cada vez con mejor capacitación y adaptación a las necesidades propias del contexto nacional e internacional (Chaves, Rojas y Chaves, 2015; Mora y León, 2012), lo que va generando una mayor apertura a buscar la calidad académica en las universidades costarricenses.

Según SINAES (2018a), en Costa Rica existen 162 carreras acreditadas con sus criterios de evaluación, las cuales han permitido, como parte de estos procesos, aplicar acciones de mejoramiento, con el fin de promover la calidad de los programas académicos que conforman estas carreras mediante la evaluación de pares externos. Mora y León (2012) indican que, para esto, SINAES ha elaborado un manual de acreditación, "el primero vigente desde el año 2000 hasta el 2009 y el segundo del 2009 hasta [la fecha, donde] se seleccionaron un conjunto de aspectos considerados relevantes en el funcionamiento de las carreras y en sus procesos de evaluación y mejoramiento continuo" (Mora y León, 2012, p. 4); entre los que están los criterios de admisibilidad, 
doi: http://dx.doi.org/10.15359/ree.22-2.16

URL: http://www.una.ac.cr/educare

CORREO: educare@una.cr

la información y promoción, el proceso de admisión e ingreso, la correspondencia con el contexto, el plan de estudios, el personal académico, el personal administrativo, la infraestructura, el centro de información y recursos, el equipo y materiales, las finanzas y presupuestos, el desarrollo docente, la metodología de aprendizaje y enseñanza, la gestión de la carrera, la investigación, entre otros parámetros que delimitan el proceso de autoevaluación de SINAES (2009).

Todos estos criterios evidencian un proceso participativo, integrador y responsable, con el único fin de fomentar, mediante la evaluación externa, el mejoramiento de las capacidades educativas de las carreras universitarias, para formar profesionales de calidad según las necesidades costarricenses y globales. Desde esta perspectiva, la evaluación externa promovida por SINAES (2009) cumple con estándares internacionales de calidad, ya que como ente nacional de evaluación se ha fortalecido y autoevaluado al generar convenios con organizaciones evaluadoras de la enseñanza universitaria en otros países, tales como Red lberoamericana para la Acreditación de la Calidad de la Educación Superior-RIACES- (UNESCO, IESALC, s. f.), la Red Internacional de Aseguramiento de la Calidad de Agencias de Educación Superior -INQAAHE, por sus siglas en inglés- (INQAAHE, 2016), entre otras, que propician mejoramiento continuo en el proceso de evaluación que se tiene en el país. Incluso, en el caso de la INQAAHE, el SINAES se ha sometido a evaluaciones por parte de este ente internacional con el fin de evidenciar la rigurosidad de la acreditación que promueve (SINAES, 2018b).

Cabe señalar, que la evaluación externa es un proceso que ha venido fortaleciendo la academia a nivel nacional e internacional, debido a que promueve la rigurosidad educativa en pro de formar profesionales integrales que ayuden al mejoramiento de la calidad de vida en el mundo. Instancias como SINAES (2009) en Costa Rica, la Agencia Nacional para la Evaluación de la Calidad y Acreditación -ANECA- (ANECA, s. f.) en España, el Consejo Nacional de Acreditación (CNA, s. f.) en Colombia, por mencionar algunos, han impulsado que la evaluación externa de carreras se convierta en una herramienta de avances académicos innovadores, y que debe convertirse en cultura no solo en nuestro país, sino también a nivel global.

\section{Conclusiones}

- La acreditación en las instituciones universitarias costarricenses, según lo indican los informes de acreditación de diferentes carreras de la UNA, ha traído beneficios en la infraestructura, en la gestión académica, en la actualización del profesorado, en la mejora de la ejecución de lineamientos, en el seguimiento al estudiantado graduado, en la revisión y actualización de los planes de estudios, entre otros aspectos.

- La acreditación es una forma de asegurar la calidad en la actualidad, donde la apertura y autonomía creciente de instituciones de nivel superior no siempre cumplen con niveles de rigurosidad en los programas ofertados para diferentes carreras. 
- La evaluación de las instituciones de educación superior en Costa Rica se rige por los parámetros establecidos por el Sistema Nacional de Acreditación de la Enseñanza Superior (SINAES).

- Los procesos de acreditación aseguran la congruencia entre la oferta educativa y la calidad de la educación superior.

- Las universidades deben garantizar la existencia de recursos adecuados, la eficacia de sus docentes y la exigencia académica.

- Para responder a las demandas de la sociedad, las instituciones de educación superior deben desarrollar estrategias culturales, científicas y tecnológicas que permitan espacios de reflexión crítica y análisis social.

- La promoción del mejoramiento continuo le permite a las universidades responder a las necesidades de desarrollo de un país y a las exigencias sociales y de conocimiento que requiere una nación para ser competitiva en un mundo globalizado.

- La cultura institucional hacia la calidad de la enseñanza universitaria es un proceso que compete desde los primeros eslabones de los centros de estudios superiores, es decir, estudiantes, docentes y personal administrativo de las carreras, hasta las autoridades.

\section{Referencias}

Agencia Nacional de Evaluación de la Calidad y Acreditación. (s. f.). Recuperado de http://www. aneca.es/ANECA

Agencia Nacional de Evaluación y Acreditación de la Educación Superior. (2014). Modelo Nacional de Evaluación y Acreditación. Mecanismo de Evaluación y Acreditación Institucional. Paraguay: Autor. http://www.aneaes.gov.py/aneaes/datos/Mecanismo/01 Mecanismo de evaluacion institucional.pdf

Águila, V. (2005). El concepto calidad en la educación universitaria: Clave para el logro de la competitividad institucional. Revista Iberoamericana de Educación, 35(5), 1-7. Recuperado de http://profefelipe.mex.t//imagesnew/7/4/6/3/6/COMPETITITVIDAD\%20 INSTITUTIONAL.pdf

Asamblea Legislativa de la República de Costa Rica. (29 de mayo de 1996). Ley 7600 sobre igualdad de oportunidades para las personas con discapacidad. Diario Oficial La Gaceta No. 102. Recuperado de http://www.documentos.una.ac.cr/bitstream/handle/unadocs/4316/ Ley 7600 Igualdad Oportunidades Personas con Discapacidad.pdf?sequence $=1$ 
doi: http://dx.doi.org/10.15359/ree.22-2.16

URL: http://www.una.ac.cr/educare

CORREO: educare@una.cr

Chaves, L. A., Rojas, D. y Chaves, O. L. (2015). El efecto de la calidad en un programa de estudios acreditados: Caso del bachillerato en la enseñanza del inglés en la Universidad Nacional, Costa Rica. Revista Calidad en la Educación Superior, 6(2), 179-199. Recuperado de http:// investiga.uned.ac.cr/revistas/index.php/revistacalidad/article/view/1125/1071

Consejo Nacional de Acreditación. (s. f.). Sistema Nacional de Acreditación en Colombia. Recuperado de https://www.cna.gov.co/1741/article-186365.html

Giraldo, U., Abad, D. y Díaz, E. (s. f.). Bases para una política de calidad de la educación superior en Colombia. Recuperado de https://www.cna.gov.co/1741/articles-186502 doc academico10.pdf

INQAAHE (2016). Recuperado de http://www.inqaahe.org/

Jara, O. (2011). La evaluación y la sistematización. $F(x)=$ Educatión Global Research, 0, 110-112. Recuperado de http://educacionglobalresearch.net/wp-content/uploads/06-Jara-1Castellano.pdf

Miranda, C. (2007). Educación superior, mecanismos de aseguramiento de la calidad y formación docente: Un debate pendiente en Chile. Estudios Pedagógicos 33(1), 95-108. doi: https:// doi.org/10.4067/S0718-07052007000100006

Mora, J. y León, G. (2012). Efectos de la acreditación en carreras universitarias seleccionadas en la educación universitaria de Costs Rica (Cuarto informe del estado de la educación, informe final). San José, Costa Rica: CONARE. Recuperado de https://estadonacion.or.cr/files/ biblioteca virtual/educacion/004/mora-y-leon-acreditacion-universitaria.pdf

OCDE. (s. f). Análisis de la OCDE acerca de las políticas nacionales para educación: La educación en Costa Rica. Recuperado de http://www.oecd.org/education/school/La-Educacion-enCosta-Rica-Resumen-Ejecutivo.pdf

Padilla-Canales, C., Brooks-Calderón, P., Jiménez-Porras, L. D. y Torres-Salas, M. I. (2015). Dimensiones de las competencias científicas esbozadas en los programas de estudio de biología, física y química de la educación diversificada y su relación con las necesidades de desarrollo científico-tecnológico de Costa Rica. Revista Electrónica Educare, 20(1), 1-26. doi: https://doi.org/10.15359/ree.20-1.2

Pedraja, L. (2013). La acreditación continúa siendo una de las más importantes medidas de calidad. Sello, 8(15), 8-11. Recuperado de https://issuu.com/santotomaschile/docs/sello julio2013 
Pires, S. y Lemaitre, M. J. (2008). Sistemas de acreditación y evaluación de la educación superior en América Latina y el Caribe (Cap. 8). En A. L. Gazzola y A. Didriksson (Eds.), Tendencias de la educación superior en América Latina y El Caribe (pp. 297-318). Caracas: IESALC-UNESCO. Recuperado de http://www.oei.es/historico/salactsi/CAPITULO 08 Pires.pdf

Scheele, J. (2009). Procesos de acreditación: Información e indicadores (Documento de trabajo CPCE N. ${ }^{\circ}$ 8), Chile: Centro de Políticas Comparadas de Educación, Universidad Diego Portales, Chile. Recuperado de https://www.cna.gov.co/1741/articles-186502 JScheele.pdf

SINAES. (2009). Manual de acreditación oficial de carreras de grado del Sistema Nacional de Acreditación de la Educación Superior. San José. Costa Rica: Autor. Recuperado de http:// derecho.ucr.ac.cr/sites/all/documentos/Acreditacion/Gestion de Calidad/Manual de Acreditaci\%C3\%B3n Sinaes.pdf

SINAES. (2015). Cinco beneficios que la acreditación ofrece a las carreras universitarias y parauniversitarias. Recuperado de http://www.sinaes.ac.cr/index.php?option=com content\&view=article\&id=76:5-razones-que-la-acreditacion-de-sinaes-ofrece-a-lascarreras-universitarias-y-parauniversitarias\&catid=8\&ltemid=112

SINAES. (2018a). Lista de carreras con acreditación oficial (Actualizada a marzo, 2018). Recuperado de https://www.sinaes.ac.cr/

SINAES. (2018b). SINAES se someterá a la evaluación de INQAAHE. Recuperado de https://www. sinaes.ac.cr/index.php/comunicados/170-sinaes-se-sometera-a-la-evaluacion-deinqaahe

Torche, P., Martínez, J., Madrid, J. y Araya. J. (2015). ¿Qué es "educación de calidad" para directores y docentes? Calidad de la Educación, 43, 103-135. Recuperado de http://www.scielo.cl/pdf/ caledu/n43/art04.pdf

Tünnermann, C. (2008). La calidad de la educación superior y su acreditación: La experiencia centroamericana. Revista da Avaliação da Educação Superior, 13(2), 313-336. doi: https:// doi.org/10.1590/S1414-40772008000200005

UNESCO. (1998). Declaración mundial sobre la educación superior en el siglo XXI: Visión y acción. París: Autor. Recuperado de http://www.unesco.org/education/educprog/wche/ declaration spa.htm\#declaracion

UNESCO. (2013). Situación educativa de América Latina y el Caribe: Hacia una educación de calidad para todos. Santiago: OREALC/UNESCO.

UNESCO, IESALC. (s. f.). Red Iberoamericana para la acreditación de la calidad de la educación superior (RIACES). Iberoamérica: Autor. Recuperado de http://www.iesalc.unesco.org.ve/ index.php?option=com fabrik\&view=details\&formid=1\&rowid=19\&lang=es 
doi: http://dx.doi.org/10.15359/ree.22-2.16

URL: http://www.una.ac.cr/educare

CORREO: educare@una.cr

Universidad Nacional, Escuela de Ciencias Biológicas. (2013). Informe de autoevaluación de la carrera Licenciatura en la Enseñanza de las Ciencias. Manuscrito inédito.

Universidad Nacional, Escuela de Ciencias Biológicas. (2014). Informe de autoevaluación de la carrera Bachillerato en Biología con énfasis: en Biología Tropical, en Biología Marina y en Biotecnología, Licenciatura en Biología con énfasis en Manejo de Recursos Naturales y en Manejo de Recursos Marinos y Dulce Acuícolas. Escuela de Ciencias Biológicas. Manuscrito inédito.

Yamada, G., Castro, J.F.y Rivera, M. (2012). Educación superioren el Perú:Retos parael aseguramiento de la calidad. Perú: SINEACE. Recuperado de http://www2.congreso.gob.pe/sicr/ cendocbib/con4 uibd.nsf/0198694D6FE2A18D05257AF5005E5B08/\$FILE/107070418Educacion-superior-en-el-Peru-Retos-para-el-aseguramiento-de-la-calidad.pdf

Zapata, G. y Tejeda, I. (2009). Impactos de aseguramiento de la calidad y acreditación de la educación superior. Consideraciones y proposiciones. Calidad en la Educación, 31, 191209. Recuperado de https://dialnet.unirioja.es/servlet/articulo?codigo $=3214471$ 